Research Article

\title{
Blood Exosomes Have Neuroprotective Effects in a Mouse Model of Parkinson's Disease
}

\author{
Ting Sun, Zhe-Xu Ding, Xin Luo, Qing-Shan Liu $\mathbb{D}$, and Yong Cheng \\ Key Laboratory for Ethnomedicine for Ministry of Education, Center on Translational Neuroscience, College of Life and \\ Environmental Sciences, School of Pharmacy, Minzu University of China, Beijing, China
}

Correspondence should be addressed to Qing-Shan Liu; nlqsh@163.com and Yong Cheng; yongcheng@muc.edu.cn

Received 25 July 2020; Revised 7 October 2020; Accepted 28 October 2020; Published 26 November 2020

Academic Editor: Kambiz Hassanzadeh

Copyright ( 92020 Ting Sun et al. This is an open access article distributed under the Creative Commons Attribution License, which permits unrestricted use, distribution, and reproduction in any medium, provided the original work is properly cited.

\begin{abstract}
Parkinson's disease (PD) is a common and complex neurodegenerative disease; the pathogenesis of which is still uncertain. Exosomes, nanosized extracellular vesicles, have been suggested to participate in the pathogenesis of PD, but their role is unknown. Here, a metabolomic analysis of serum and brain exosomes showed differentially expressed metabolites between 1 Methyl-4-phenyl-1, 2, 3, 6-tetrahydropyridine hydrochloride- (MPTP-) induced PD mice and control mice, such as oxidized lipids, vitamins, and cholesterol. These metabolites were enriched in coenzyme, nicotinamide, and amino acid pathways related to PD, and they could be served as preclinical biomarkers. We further found that blood-derived exosomes from healthy volunteers alleviated impaired motor coordination in MPTP-treated mice. Results from immunohistochemistry and western blotting indicated that the loss of dopaminergic neurons in substantia nigra and striatum of PD model mice was rescued by the exosome treatment. The exosome treatment also restored the homeostasis of oxidative stress, neuroinflammation, and cell apoptosis in the model mice. These results suggest that exosomes are important mediators for PD pathogenesis, and exosomes are promising targets for the diagnosis and treatment of PD.
\end{abstract}

\section{Introduction}

Parkinson's disease (PD) is the second most common neurodegenerative disease, affecting approximately $1 \%$ of the population aged $>60$ years $[1,2]$. A meta-analysis of the global data indicated the rising prevalence of PD with age along with some differences in prevalence by geographic location and sex $[3,4]$. The first detailed description of PD was made approximately two centuries ago, but the pathogenesis of the disease remains unclear [5]. Its cardinal motor symptoms are bradykinesia, tremor, rigidity, flexed posture, postural instability, and freezing of gait $[6,7]$. The crucial pathological feature of PD is the degeneration of dopaminergic neurons in the substantia nigra pars compacta $(\mathrm{SNpc})$, a reduction in dopamine content in the striatum $(\mathrm{Cpu})$, and the appearance of Lewy bodies $[5,6]$, which are aggregated by abnormally folded $\alpha$-synuclein. Misfolded $\alpha$-synuclein is the most abundant protein inclusion that can categorize PD with other neurodegenerative diseases. PD results from a complicated interplay of genetic and environmental factors, such as cigarette smoking and caffeine [8-10]. However, we cannot treat PD by modifying genetic or environmental risk factors. Neuroinflammation also contributes to PD pathology. In the early stages of diseases, astrocytes and microglia are both involved in the clearance of extracellular debris or release of nutrients and anti-inflammatory factors, which might aid the survival of neurons $[11,12]$. However, activated microglia can release harmful reactive oxygen species, nitrogen species, and proinflammatory cytokines [13, 14]. Prolonged overactivation of microglia will exacerbate the death of dopaminergic neurons in the nigra $[15,16]$. Accumulating evidence indicates that oxidative stress plays a key role in all forms of PD [17]. Studies of young PD patients have revealed that elevated oxidative stress is an important trait of the early disease stages and occurs before severe neuronal loss [18]. Exosomes, the smallest extracellular vesicles (diameter range $50-150 \mathrm{~nm}$ ), are released by the fusion of multivesicular endosomal bodies with the plasma membrane $[19,20]$. 
Exosomes can be released both in vitro and in vivo from a variety of nerve cells, such as neurons [21], microglia [22], and astrocytes [23]. Exosomes carry noncoding RNA such as miRNA, lncRNA, and circRNA, along with proteins and lipids [24, 25], and mediate intercellular and interorgan communication. In PD, exosomes secreted by neurons have been found to carry pathogenic proteins such as $\alpha$-synuclein and are transmitted to other neurons or glial cells, leading to the further spread of misfolded $\alpha$-synuclein and the occurrence of neuroinflammation [26-28]. There is reason to believe that the release and transmission of exosomes can dynamically reflect the pathological changes in cells in inaccessible areas such as the brain [29]. Some studies have demonstrated that the expression of proteins and RNAs in exosomes derived from the sera and cerebrospinal fluid (CSF) of PD patients significantly differ from those of healthy people [30]. The studies by Shi et al. [31] and Stuendl et al. [32] found significant differences in the exosomal $\alpha$-synuclein in the plasma and CSF exosomes in PD patients and controls. The study also found a large increase in the number of exosomes in the CSF of PD patients [32]. Therefore, exosome has been suggested as a promising target for the diagnosis of PD [33]. Given the close relationship between dopamine loss and the metabolism of amino acids such as tyrosine, exosomal metabolomics analysis could receive more attention for predicting disease or discovering new potential biomarkers. However, there are no detailed studies on exosomal metabolites in relation to PD. In addition, signal molecules carried by exosomes may also affect gene expression in target cells. Many studies have indicated that exosomes derived from multiple cells may have therapeutic effects in neurological diseases such as stroke [34, 35], ischemiareperfusion injury [36], traumatic brain injury [37, 38], and Alzheimer's disease [39]. Our latest study found that serum exosomes from major depressive disorder patients caused depressive-like behavior in healthy mice, and serum exosomes from healthy volunteers alleviated the depressive-like behaviors in unpredictable mild stress-treated mice [40]. We hypothesize that exosomes may also have potential predictive and therapeutic effects in PD. In this study, we are aimed at utilizing widely-targeted metabolomics technology to analyze the serum and brain exosomal metabolite differences between healthy and 1-Methyl-4-phenyl-1, 2, 3, 6tetrahydropyridine hydrochloride- (MPTP-) induced PD mice, with the aim of identifying potential biomarkers of PD. In addition, we are aimed at exploring whether "normal" exosomes protect against PD.

\section{Materials and Methods}

2.1. Preparation of Exosomes. All volunteers were recruited from Minzu University of China (Supplementary Table 1). The blood exosome isolation and validation methods were performed as previously reported [41]. Briefly, exosomes were isolated from the diluted blood using a $\mathrm{qEV}$ column and concentrated with a $30 \mathrm{~K}$ MWCO PES protein concentrator-Vivaspin ${ }^{\circledR}$ (Sartorius, Gottingen, Germany). The concentrated exosomes were then resuspended in $200 \mu \mathrm{l}$ phosphate-buffered saline (PBS) for further analysis.
2.2. Widely-Targeted Metabolomics. Widely-targeted metabolomics detection and quantification were conducted by the metabolomics provider METWARE on serum and brain samples from healthy control and MPTP-induced PD mice. Serum and brain exosome isolation was performed as described previously $[41,42]$. Detailed methods are provided in Supplementary file 1.

2.3. Animals and Treatment. Ten-week-old male C57BL/6 mice $(25 \pm 2 \mathrm{~g})$ were obtained from Vital River Laboratory (Beijing, China) and were housed at $24 \pm 1^{\circ} \mathrm{C}$ and $50 \pm 1 \%$ humidity under a $12 \mathrm{~h}$ light/dark cycle and provided with ad libitum access to a standard diet and drinking water. Animal procedures (including the euthanization method) were approved by the Animal Care and Use Committee of the Minzu University of China (ECMUC2019001AO). MPTP (Macklin, Shanghai, China) was used to generate the PD model. The mice were randomly divided into three groups: the control, MPTP, and exosomes+MPTP groups. The mice in the control group were intraperitoneally injected with saline. The MPTP and exosomes+MPTP groups were injected with MPTP (20 mg/kg, dissolved in saline) every $2 \mathrm{~h}$ for a total of four doses over an $8 \mathrm{~h}$ period in 1 day. The mice in the exosomes+MPTP group were caudal vein injected with exosomes $(0.2 \mathrm{ml}$ per mouse). The first injection was delivered $1 \mathrm{~h}$ before the first injection of MPTP, and thereafter once every 3 days for a total of four injections.

2.4. Behavior Test. The rotarod test was used to assess neurological impairment such as motor coordination and balance and was performed on days 1 and 11 after MPTP injection. The protocol followed was as previously reported [43]. Before MPTP injection, mice were pretrained for 3 days at $10 \mathrm{rpm}$ until they were able to remain on the rod for at least $90 \mathrm{~s}$. For the formal test, a rotating drum was accelerated from 4 to $40 \mathrm{rpm}$ over $5 \mathrm{~min}$. Each trial continued until the mice were unable to remain on the rod without falling off. The latency for the mice to fall from the rotarod was recorded $(n=3)$.

2.5. Brain Immunohistochemistry. After the rotarod test, mice were euthanized with pentobarbitone and perfused with saline. The brains were removed, postfixed in $4 \%$ paraformaldehyde overnight at $4^{\circ} \mathrm{C}$, and then soaked in $20 \%$ and $30 \%$ sucrose and processed for cryoprotection. The frozen brains were cut into $40 \mu \mathrm{m}$ coronal sections using a freezing microtome and stored in cryoprotectant (30\% ethylene glycol, 30\% sucrose, and $0.02 \mathrm{MPB}$ ) at $-20^{\circ} \mathrm{C}$ until use. Brain sections were washed in PBS and treated with $3 \% \mathrm{H}_{2} \mathrm{O}_{2}$, then blocked and perforated with $10 \%$ goat serum and $0.3 \%$ Triton $\mathrm{X}-100$. The sections were then incubated overnight with primary antibodies TH (CST, Boston, MA, USA) at $4^{\circ} \mathrm{C}$, followed by incubation with secondary antibodies which were goat antirabbit $(1: 400)$ for $1 \mathrm{~h}$ at $25^{\circ} \mathrm{C}$. Sections were mounted on gelatin-coated slides, then dried, dehydrated, and coverslipped. The histological images were examined using a bright-field microscope.

2.6. Western Blot Analysis. The substantia nigra and striatum were isolated from the brain and homogenized with RIPA buffer. The protein concentration of the lysates was 
determined by a BCA Protein Assay Kit and mixed with $4 \times$ loading buffer and boiled at $95^{\circ} \mathrm{C}$ for $5 \mathrm{~min}$. Proteins were separated by sodium dodecyl sulfate-polyacrylamide gel electrophoresis and then transferred to a nitrocellulose membrane. The percentage of the SDS-PAGE gel was $10 \%$ for tyrosine hydroxylase (TH) and actin and 12\% for Bax and Bcl-2. The blotted membrane was blocked with 5\% skim milk for $1 \mathrm{~h}$ and then incubated overnight with corresponding primary antibodies at $4^{\circ} \mathrm{C}$, followed by incubation with corresponding secondary antibodies for $1 \mathrm{~h}$ at $25^{\circ} \mathrm{C}$, and subsequently visualized with an enhanced chemiluminescence reagent. The secondary antibodies were goat antirabbit $(1: 10,000)$ and goat anti-mouse $(1: 10,000)$ from CST.

2.7. Quantitative Real-Time Polymerase Chain Reaction. Total RNA from substantia nigra and striatum was, respectively, extracted with Trizol reagent. A PrimeScript RT reagent kit was used to synthesize first-strand cDNA. Then cDNAs were quantified by real-time polymerase chain reaction on LightCycler ${ }^{\circledR} 96$ (Roche). GAPDH was used as a reference gene for analysis. Primers were synthesized by Sangon Biotech (Shanghai) Co., Ltd.

2.8. Oxidative Stress Marker Level/Activity Measurement. The MDA level and total SOD activity in the serum were measured by a colorimetric assay kit (Jiancheng Bioengineering Institute, China) according to the manufacturer's instructions.

2.9. Statistical Analysis. Data are presented as means \pm standard deviation. Statistical significance $(p<0.05)$ was analyzed by one-way ANOVA or t-test with GraphPad Prism 7.

\section{Results}

3.1. Differentially Expressed Metabolites between Control and MPTP Mice. A widely-targeted metabolomics analysis was used to evaluate the differences in exosomal metabolites in the serum and the brain in healthy control and MPTPinduced PD mice. In this study, a total of 433 metabolites were detected in four groups: serum control (Sc), serum MPTP (Sm), brain control (Bc), and brain MPTP (Bm). A Score OPLS-DA Plot and an OPLS-DA S-Plot of Bc vs. Bm and $\mathrm{Sc}$ vs. Sm are presented in Figure 1. The metabolic data were analyzed according to the OPLS-DA model, and the Scores OPLS-DA Plot was drawn to illustrate the previous differences in each component.

The Variable Importance in Projection (VIP) of the OPLS-DA model and $p$ value as determined by a MannWhitney $U$-test were used to screen the differential expression of metabolites. A VIP of $\geq 1$ and $p$ value of $<0.05$ were considered to indicate differentially expressed metabolites. The results of the differential metabolism screening are provided in Supplementary Table 1. In the serum samples, 69 metabolites were found to be differentially expressed between the control group and MPTP group. In the brain tissue, 148 metabolites were found to be differentially expressed (Supplementary Tables 2 and 3). Additionally, 25 differently expressed metabolites were found in both serum and brain tissue (Table 1).
We next used the KEGG database to annotate the differentially expressed metabolites for potential biological functions. According to the KEGG pathway enrichment diagram, pathways such as the tyrosine metabolic pathway, purine metabolism, and glutamate metabolism pathways were found to be significantly enriched for differentially expressed metabolites in the serum and brain tissue (Figure 1).

3.2. Serum Exosomal Metabolites as Potential Biomarkers for $P D$. Receiver operating characteristic (ROC) curves were utilized to evaluate the accuracy of the metabolites in the serum for potentially differentiating MPTP mice from controls. We performed ROC curve analysis using the nine metabolites that contributed most to the differentiation of MPTP mice and controls. As indicated in Figure 2, serum exosomal phenylacetate (Figure 2(a), area under the curve $[$ AUC] $=91.43 \%$ , 95\% confidence interval [CI]: 79.16-100\%), (S)-(-)-2-hydroxyisocaproic acid (Figure 2(b), AUC $=100 \%, 95 \%$ CI:100$100 \%$ ), D-glucono-1,5-lactone (Figure 2(c), AUC = 92.14\%, 95\% CI: 81.74-100\%), cholesterol (Figure 2(d), AUC= 97.86\%, 95\% CI: 93.29-100\%), triethyl phosphate (Figure 2(e), AUC = 90\%, 95\% CI: 76.29-100\%), 1,2-dichloroethane (Figure 2(f), AUC $=100 \%, 95 \%$ CI: 100-100\%), carene (Figure 2(g), AUC = 85.71\%, 95\% CI: 70.16-100\%), 2,4,6trimethylphenol (Figure 2(h), AUC $=92.86 \%$, 95\% CI: 82.92$100 \%$ ), and terpinolene (Figure 2(i), AUC $=95.71 \%$, 95\% CI: $88.36-100 \%)$ were found to have good to excellent performance to discriminate between MPTP mice and controls. This suggests that the above nine metabolites are likely to be potential biomarkers for PD.

3.3. Effects of Blood-Derived Exosomes from Healthy Volunteers on Motor Ability in PD Model Mice. The rotarod test was used to evaluate the motor coordination and balance of mice. The experimental design is illustrated in Figure 3(a). After pretraining and before MPTP injection, there was no significant difference in the latency of the mice in each group, indicating that the motor capacity of the mice in each group was similar (Figure 3(b)). On day 11, the rotarod test results indicated that MPTP treatment significantly decreased the latency and exosome treatment significantly improved the latency (Figure 3(c)) $(p<0.001)$.

3.4. Effects of Blood-Derived Exosomes from Healthy Volunteers on Dopamine Neurons in MPTP Mice. The improvement in motor dysfunction in exosome-treated MPTP-treated mice led us to hypothesize that exosomes may protect dopaminergic neurons from MPTP-induced injury. To test this hypothesis, we assessed whether exosomes prevent MPTP-induced neuronal damage. $\mathrm{TH}$ is a ratelimiting enzyme in dopamine biosynthesis and a wellknown marker of dopaminergic neurons. The level of $\mathrm{TH}$ positive neurons in the $\mathrm{SNpc}$ and $\mathrm{CPu}$ is recognized as an indicator of the severity of dopaminergic neuronal damage in MPTP-injured animals.

The immunohistochemical results in the SNpc indicated that the number of $\mathrm{TH}$-positive neurons was significantly reduced in the MPTP group compared to that in the control 

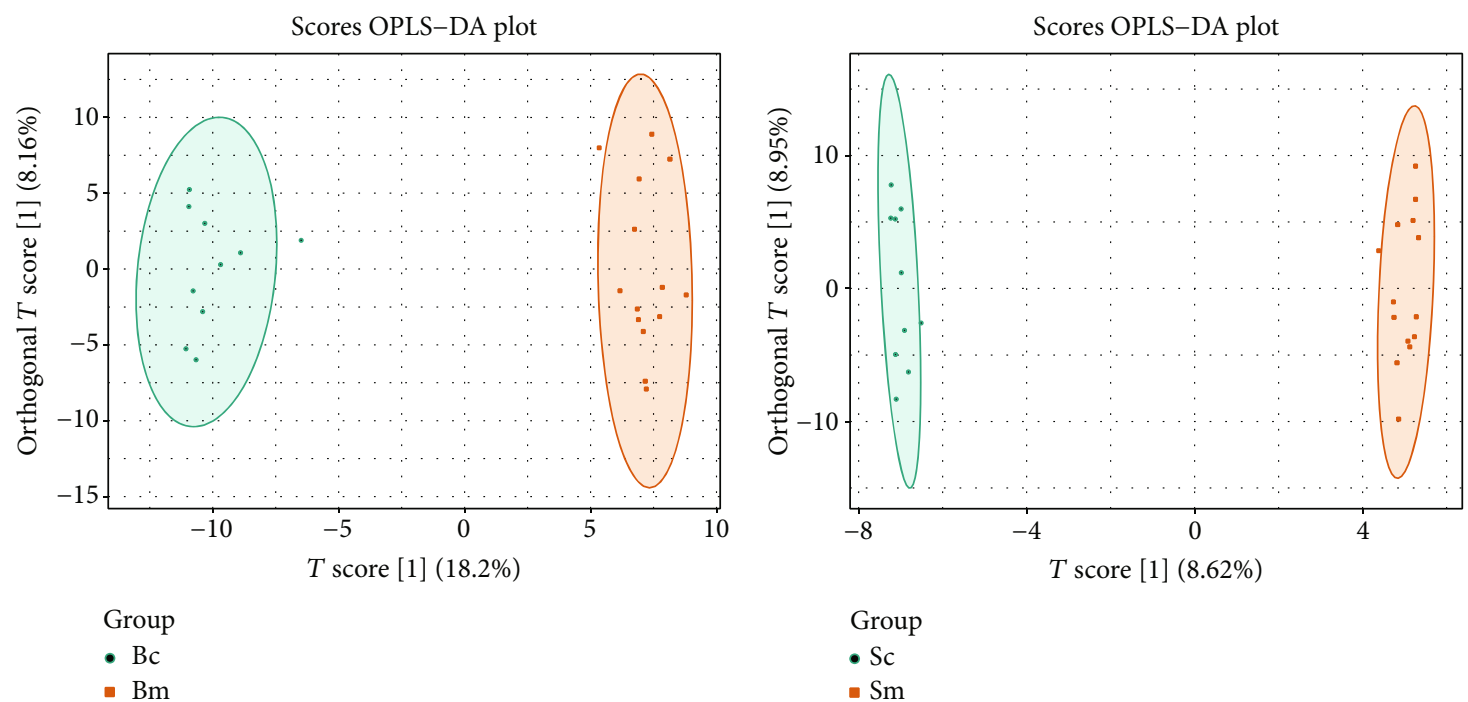

(a)

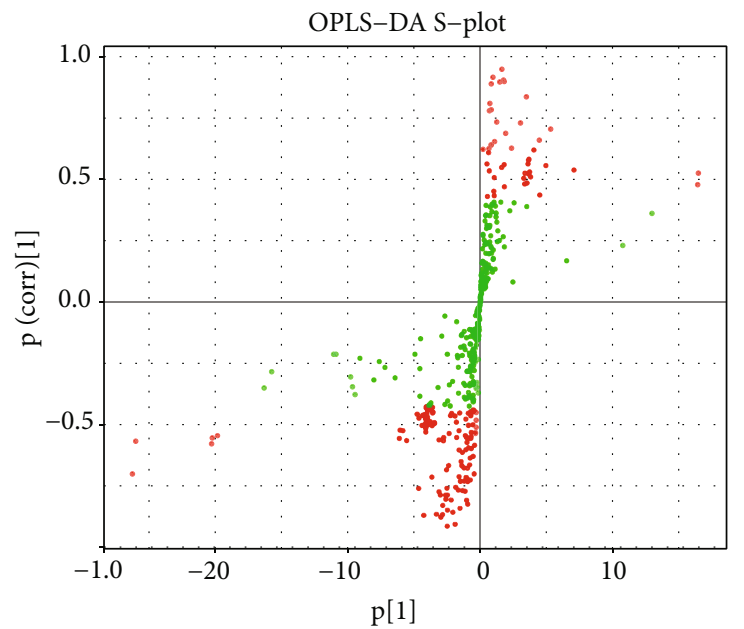

(c)

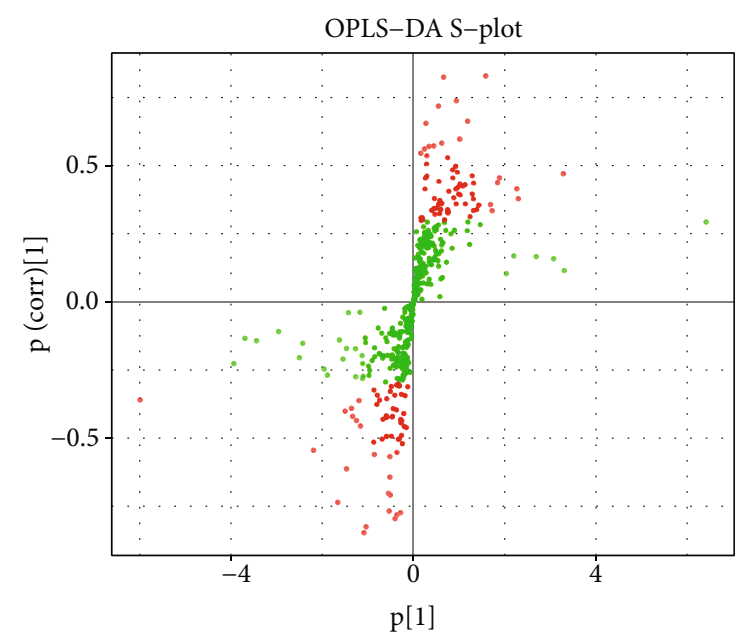

(d)
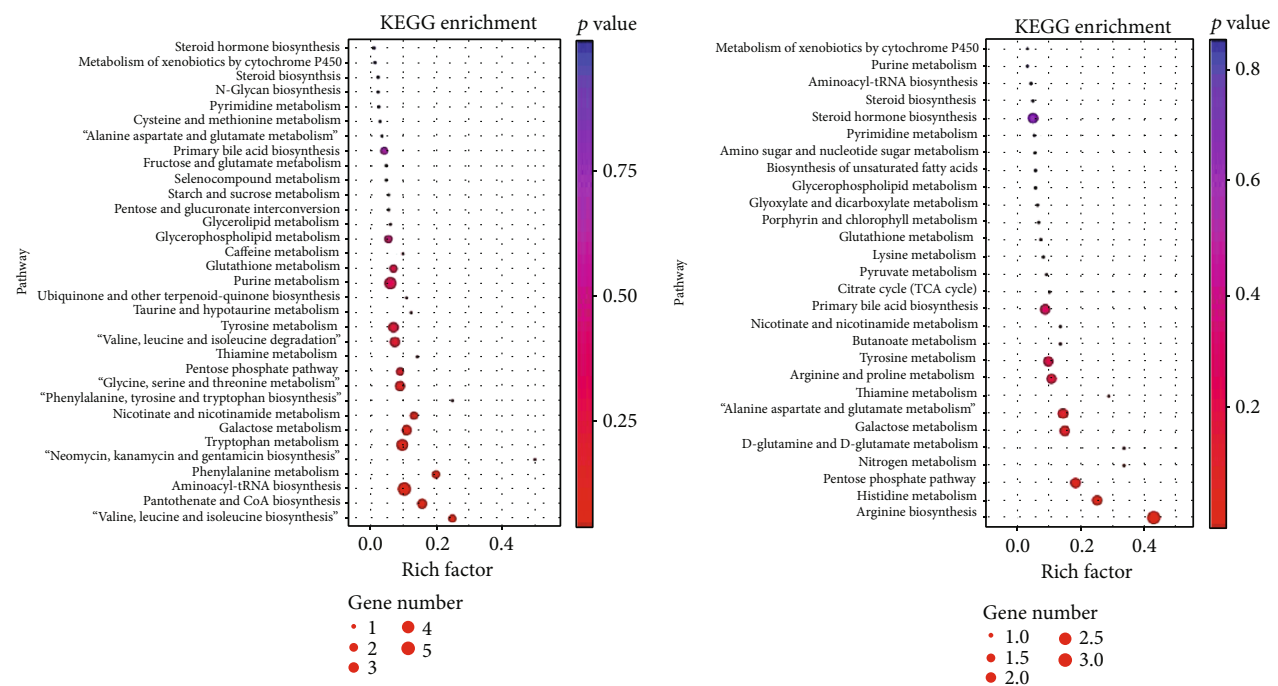

(e)

(f)

FIGURE 1: Widely-targeted metabolomics analysis was used to evaluate the differences of metabolites in the brain between control and PD model mice. Analysis of 14 Parkinson's disease mice and 10 control mice. A Score OPLS-DA Plot of metabolites in the brain (a) and serum (b). An OPLS-DA S-Plot of metabolites in the brain (c) and serum (d). Statistics of KEGG Enrichment in the brain (e) and serum (f). 
TABLE 1: 25 differentially expressed metabolites in serum and brain tissue.

\begin{tabular}{|c|c|c|c|c|c|c|c|}
\hline Compounds & Class & VIP & $\begin{array}{l}\text { Serum } \\
p \text { value }\end{array}$ & Trend & VIP & $\begin{array}{l}\text { Brain } \\
p \text { value }\end{array}$ & Trend \\
\hline (5-L-Glutamyl)-L-amino acid & Amino acid metabolomics & 1.50329 & 0.0308 & $\mathrm{Up}$ & 1.016439 & 0.0183 & $\mathrm{Up}$ \\
\hline Dulcitol & Carbohydrate metabolomics & 1.508731 & 0.0283 & Down & 1.931532 & $<0.0001$ & Down \\
\hline Phenyllactate (Pla) & $\begin{array}{l}\text { Organic acid and its } \\
\text { derivatives }\end{array}$ & 2.503092 & $<0.0001$ & Down & 1.087977 & 0.0039 & Down \\
\hline $\begin{array}{l}\text { 9,10-DiHOME }[( \pm) 9,10 \text {-dihydroxy-12Z- } \\
\text { octadecenoic acid] }\end{array}$ & Oxidized lipid & 1.826174 & 0.0067 & Up & 1.367845 & 0.0027 & Down \\
\hline Cis-11,14,17-eicosatrienoic acid (C20:3) & Lipids fatty acids & 1.56911 & 0.0203 & Up & 2.024657 & $<0.0001$ & Down \\
\hline (S)-(-)-2-Hydroxyisocaproic acid & $\begin{array}{l}\text { Organic acid and its } \\
\text { derivatives }\end{array}$ & 2.884665 & $<0.0001$ & Down & 1.71456 & 0.0002 & Down \\
\hline Neopterin & Pteridines and derivatives & 1.910464 & 0.005 & Up & 2.007469 & $<0.0001$ & Down \\
\hline 4-Ethylbenzoic acid & $\begin{array}{l}\text { Benzene and substituted } \\
\text { derivatives }\end{array}$ & 1.945169 & 0.0041 & Up & 1.970055 & $<0.0001$ & Down \\
\hline 2-Picolinic acid & $\begin{array}{c}\text { Pyridine and pyridine } \\
\text { derivatives }\end{array}$ & 1.332943 & 0.0481 & Down & 1.325756 & 0.0032 & Down \\
\hline Adenine & Nucleotide metabolomics & 1.548782 & 0.0288 & Down & 1.323111 & 0.0064 & Down \\
\hline D-Glucono-1,5-lactone & Carbohydrate metabolomics & 2.446655 & $<0.0001$ & Up & 1.465692 & 0.0013 & Up \\
\hline Nicotinic acid & $\begin{array}{c}\text { CoOthersEnzyme factor \& } \\
\text { vitamin }\end{array}$ & 1.906224 & 0.0066 & Down & 1.084725 & 0.0124 & Down \\
\hline 1-Naphthylacetic acid & $\begin{array}{l}\text { Organic acid and its } \\
\text { derivatives }\end{array}$ & 1.490897 & 0.0181 & Down & 1.612702 & 0.0008 & Up \\
\hline Cholesterol & Lipids & 2.80908 & $<0.0001$ & Up & 2.226335 & $<0.0001$ & Up \\
\hline Hexadecanamide & Lipids fatty acids & 1.291087 & 0.0274 & Up & 1.304757 & 0.0301 & Up \\
\hline Triethyl phosphate & $\begin{array}{l}\text { Organic acid and its } \\
\text { derivatives }\end{array}$ & 2.413266 & 0.0001 & Down & 2.140886 & $<0.0001$ & Down \\
\hline 1,2-Dichloroethane & Hydrocarbon derivative & 2.825226 & $<0.0001$ & Up & 1.319369 & 0.0062 & Up \\
\hline (E)-2-Octen-1-ol & Alcohol & 1.769306 & 0.0077 & Down & 1.246376 & 0.0071 & Down \\
\hline Barbituric acid & Heterocyclic compound & 1.554395 & 0.0277 & Down & 1.196766 & 0.0072 & Down \\
\hline Carene & Heterocyclic compound & 2.232867 & 0.0004 & Up & 2.123199 & $<0.0001$ & Down \\
\hline Hexyl acetate & Fatty acyls & 1.79975 & 0.0087 & Up & 1.554381 & 0.0003 & Down \\
\hline 2,4,6-Trimethylphenol & Phenols and its derivatives & 2.660509 & $<0.0001$ & Down & 1.641595 & 0.0002 & Down \\
\hline Terpinolene & Terpenoid & 2.707682 & $<0.0001$ & Down & 1.474065 & 0.0004 & Down \\
\hline Octanal & Aldehyde & 1.481981 & 0.0278 & Down & 1.129016 & 0.0196 & Down \\
\hline Naphthalene & $\begin{array}{l}\text { Benzene and substituted } \\
\text { derivatives }\end{array}$ & 1.663639 & 0.0121 & Down & 1.255286 & 0.0057 & Down \\
\hline
\end{tabular}

The name of compounds, class, VIP, $p$ value, and trend of metabolites were shown in the table.

group $(p<0.0001)$. Exosome treatment almost completely reversed this reduction $(p<0.0001$ ) (Figures $4(\mathrm{a})$ and $4(\mathrm{~b})$ ). Similar results were also reflected in the immunohistochemical staining of the $\mathrm{CPu}$ (Figure $4(\mathrm{c})$ ). To further confirm these findings, we measured $\mathrm{TH}$ protein levels in the SNpc and $\mathrm{CPu}$ by western blot. Compared to the control group, TH protein expression was significantly reduced in both the SNpc and the CPu in the MPTP group $(p<0.0001)$, whereas exosome treatment significantly alleviated this reduction $(p<0.05)$ (Figures $4(\mathrm{~d})$ and $4(\mathrm{e}))$.

3.5. mRNA Levels of Inflammatory and Anti-Inflammatory Factors in the SNpc and CPu. Neuroinflammation is one of the key pathogenic features of PD. In this study, we examined inflammatory and anti-inflammatory cytokine mRNA expression in the $\mathrm{SNpc}$ and $\mathrm{CPu}$. The results revealed that the mRNA levels of interleukin (IL)-1 $\beta$, IL-6, and tumor necrosis factor- (TNF-) $\alpha$ were significantly increased and those of IL-4, IL-10, and transforming growth factor(TGF-) $\beta$ were significantly decreased by MPTP treatment in the SNpc $(p<0.05)$. Exosome treatment significantly reduced the mRNA levels of IL1 $\beta$, IL- 6 , and TNF- $\alpha$ and improved the mRNA levels of IL-4, IL-10, and TGF- $\beta$ $(p<0.05)$ (Figures 5(a)-5(f)). Similar changes in IL-1 $\beta$, IL6 , IL-4, IL-10, and TGF- $\beta$ were observed in the $\mathrm{CPu}$ $(p<0.05)$ (Figures 5(g)-5(l)). These results indicate that neuroinflammation caused by MPTP could be relieved by exosome treatment.

3.6. Effects of Blood-Derived Exosomes from Healthy Volunteers on Oxidative Stress and Apoptosis. Oxidative stress has been implicated in the etiology of PD. The serum 


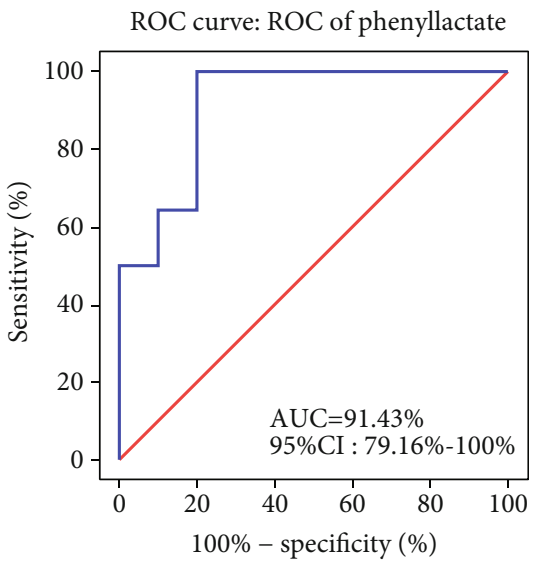

(a)

ROC curve:

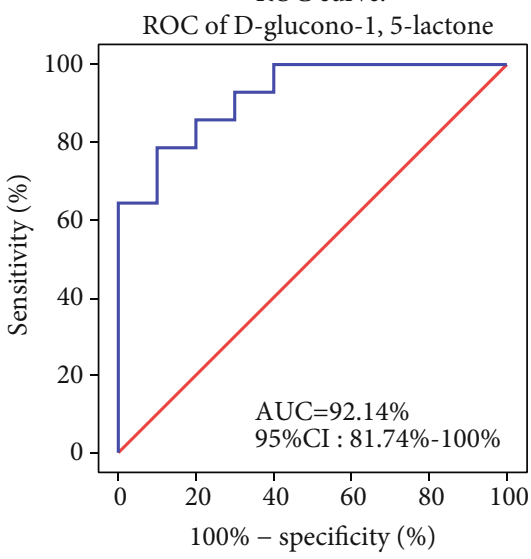

(c)

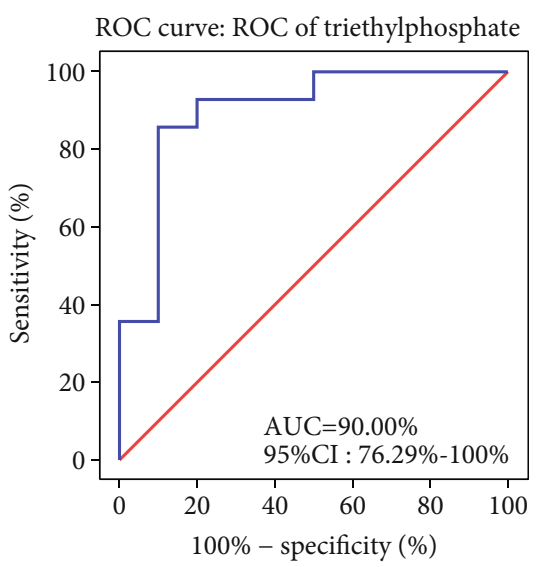

(e)
ROC curve:

ROC of (S)-(-)-2-hydroxyisocaproic acid

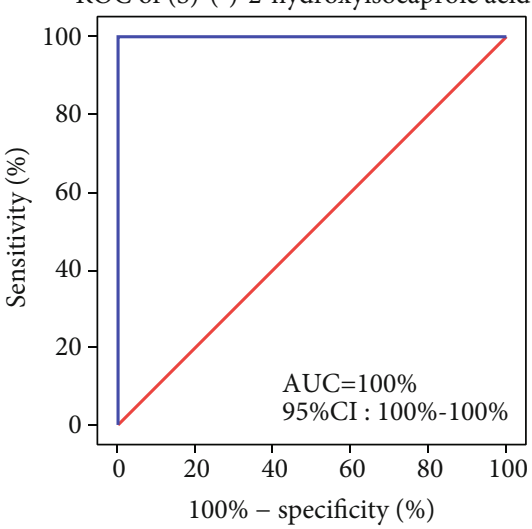

(b)

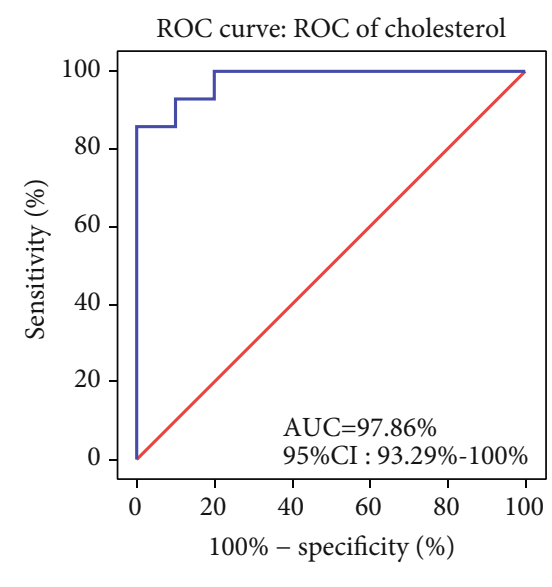

(d)

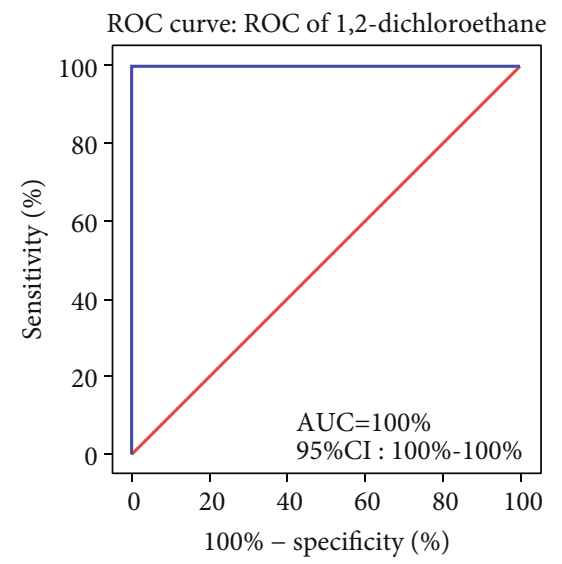

(f)

Figure 2: Continued. 


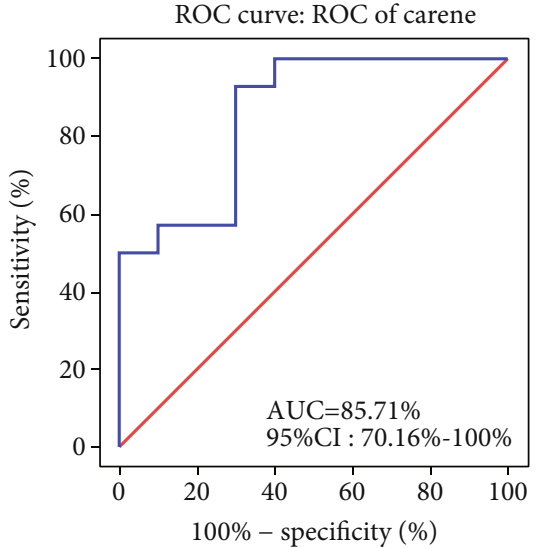

(g)

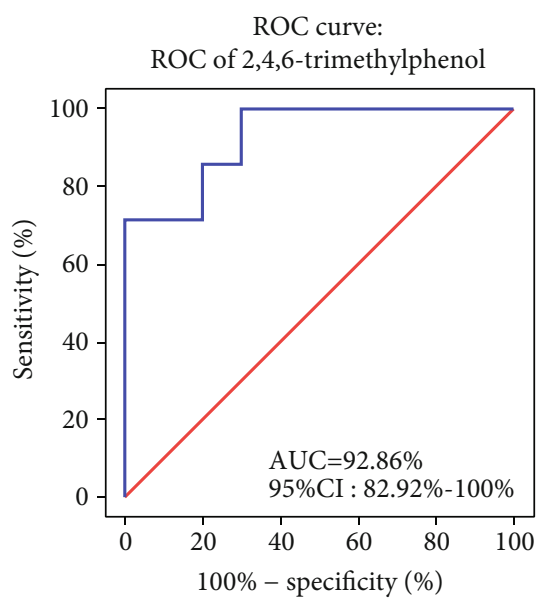

(h)

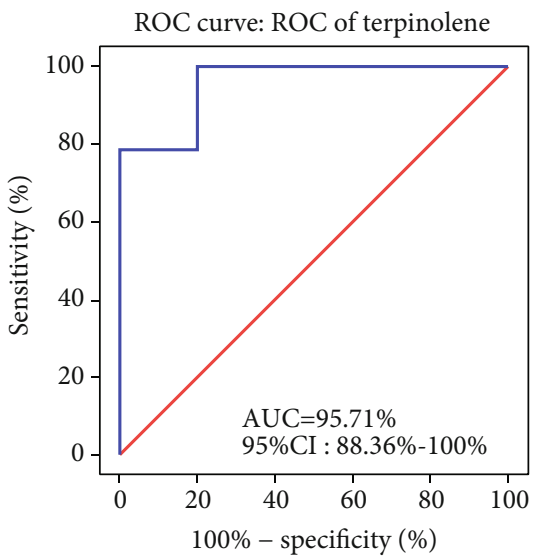

(i)

Figure 2: Serum exosomal metabolites as biomarkers for PD. ROC curves of Phenyllactate (a), (S)-(-)-2-hydroxyisocaproic acid (b), Ddlucono-1,5-lactone (c), cholesterol (d), triethyl phosphate (e), 1,2-dichloroethane (f), carene (g), 2,4,6-trimethylphenol (h), and Terpinolene (i) in serum exosomes of MPTP mice and controls. Results were analyzed with 10 controls and 14 MPTP mice.

malondialdehyde (MDA) and superoxide dismutase (SOD) levels were measured to assess the degree of oxidative stress injury and antioxidative ability. The results indicated that, compared to the control condition, MPTP treatment significantly increased the MDA level, and exosomes markedly alleviated this change $(p<0.05)$ (Figure 6(a)). Similarly, the SOD level was significantly reduced by MPTP but increased by exosomes $(p<0.05)$ (Figure 6(b)). In addition, Bcl-2 and Bax were detected, which reflected antiapoptosis and apoptosis in the brain, respectively. The results indicated a significant reduction in Bcl-2 protein expression but an increase in Bax expression with MPTP treatment $(p<0.05)$. Exosomes also upregulated Bcl-2 and downregulated Bax expression $(p<0.05)$. The above results were consistent in the SNpc (Figures 6(c) and 6(e)) and $\mathrm{CPu}$ (Figures 6(d) and 6(f)).

\section{Discussion}

The etiology of PD is complex and diverse and involves the interaction of genetic factors and the external environment. The occurrence of the disease is often associated with imbalances in neurotransmitters, lipid and energy metabolism disorders, and mitochondrial dysfunction. Neurotransmitters play a role in signal transduction via metabolic pathways such as release and reuptake. Changes in any of these metabolic pathways may affect central nervous system function. Small changes in endogenous and exogenous factors can be reflected in metabolite levels. Based on metabolomics approaches, metabolites associated with dopamine, purines, amino acids, fatty acids, and polyamines were found to be highly correlated with the progression of PD $[44,45]$. Most of such studies are based on CSF and blood analysis, although some have examined other biological samples such as urine, feces, or brain tissue [46]. However, blood (serum or plasma) metabolites do not fully reflect the metabolism in the brain, and CSF samples are difficult to acquire. Therefore, exosomes, which can cross the blood-brain barrier, were used in this study to synchronously assess the metabolic changes in both the brain and the serum. Our results revealed a close relationship between exosome metabolites and PD. In this study, we found significant differences in 13 amino acids and metabolites in brain exosomes. Tyrosine and phenylalanine, which are closely involved in dopamine metabolism, were significantly downregulated in the PD group compared to the control group. Tyrosine is taken up by dopaminergic neurons and catalyzed by tyrosine hydroxylase in the 


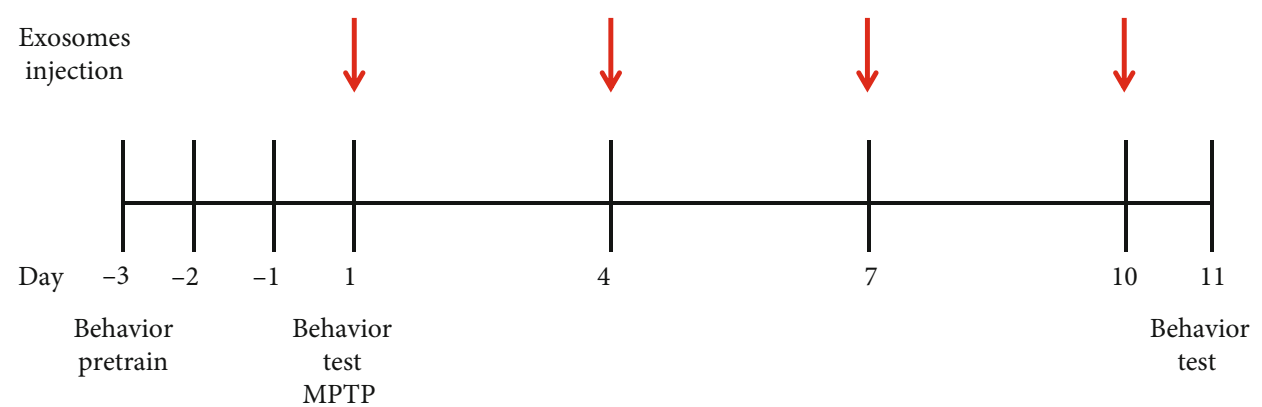

(a)

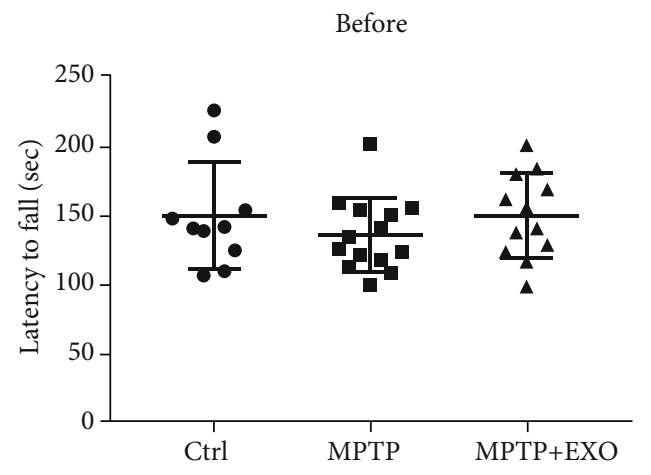

(b)



(c)

Figure 3: Exosomes improve behavioral performances in MPTP-induced mice. Protocol for MPTP administration, exosome administration, and behavioral test in Parkinson's disease model mice (a). Rotarod test for mice before MPTP injection (b), and after MPTP injection (c). All values are means $\pm \mathrm{SD}, n=10-14,{ }^{* *} p<0.01$, and ${ }^{* * *} p<0.001$.

cytoplasm to L-dopa, which is subsequently decarboxylated to dopamine. Phenylalanine also forms tyrosine via phenylalanine hydroxylase. The reduction of tyrosine and phenylalanine reflects the reduction of dopamine, which is one of the most important mechanisms in PD. The decrease in dopamine results in relative hyperfunction of the acetylcholine system. This transmitter disorder is the key to dyskinesia and other abnormal neurological activities. Our findings indicated that in PD mice, glutamic acid, glutamine, and glutamate were significantly reduced in serum exosomes; branched-chain amino acids (leucine, isoleucine, and valine) were reduced in brain exosomes; and alanine was increased in both serum and brain exosomes. Notably, there were significant differences in many lipids and fatty acids in both serum and brain exosomes between PD and control mice. The findings are similar to those of previous studies reporting that these metabolism pathway alterations are all indicative of mitochondrial dysfunction $[44,47]$. In addition, decreased adenine in both serum and brain exosomes may be related to their participation in biological oxidation reactions. Uric acid, the final product of purine metabolism, can remove reactive oxygen and reactive nitrogen and reduce oxidative/nitrative stress. These results confirmed that the metabolism of alanine, purine, and branched amino acids and lipids, which are closely involved in mitochondrial function and biological redox homeostasis, play an important role in the progression of PD. Moreover, the metabolites of exosomes were also indicative of alterations to carbohydrate metabolomics pathways. The serum and brain exosomes of PD mice accordantly exhibited a significant decrease in dulcitol and an increase in D-glucono-1, 5-lactone. In brain exosomes, phosphate and glucose were significantly reduced in PD mice. In serum exosomes, galactose and galactopyranoside were significantly increased in PD mice. It is not yet clear why these trends differ, but it may reflect the differential release of exosomes from different parts of the body. These results were consistent with previous results in body fluids [44]. The KEGG database revealed the differential metabolic pathways of phenylalanine and tyrosine metabolism, tryptophan metabolism, purine metabolism, branched-chain amino acid metabolism (leucine, isoleucine, and valine), etc. Other previous studies [48-51] also reported changes in purine metabolism, oxidative stress/redox homeostasis, energy metabolism, fatty acid metabolism, branched chain amino acids, phenylalanine and tyrosine metabolism, tryptophan metabolism, glycine derivation, and steroidogenesis in body fluids.

Growing evidence indicates that exosomes contribute to the progression of neurodegenerative disease [52]. Ghidoni et al. proposed the "Trojan horse" hypothesis of exosomes in neurodegeneration, a mechanism leading to the death of cells by shipping toxic agents in exosomes from cell to cell [53]. Indeed, many researchers believe that exosomes act as potential intercellular carriers of pathogenic proteins such as $\alpha$-synuclein and cause impaired neuronal function [54]. In fact, a recent study by Han et al. showed that mice treated with serum exosomes from PD patients exhibited PDrelevant molecular, cellular, and behavioral phenotypes [55]. However, the opposing view is also considered feasible, that is, exosomes also offer neuroprotection, altering the 


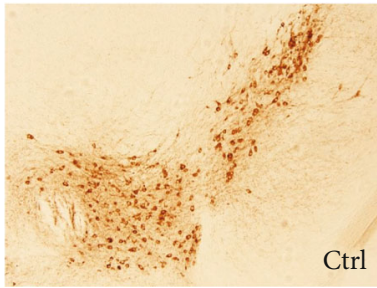

Ctrl

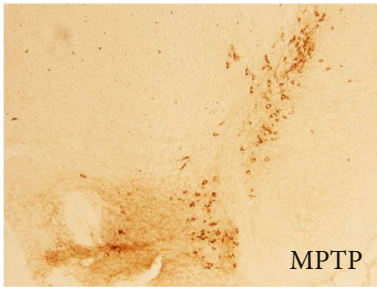

(a)

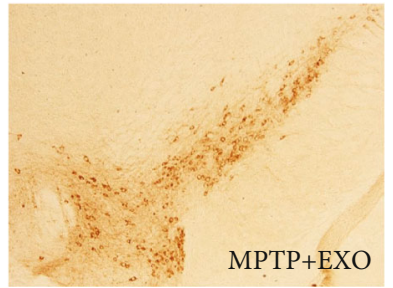

Ctrl
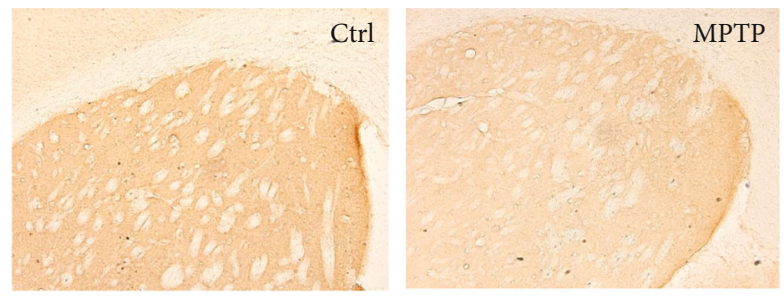

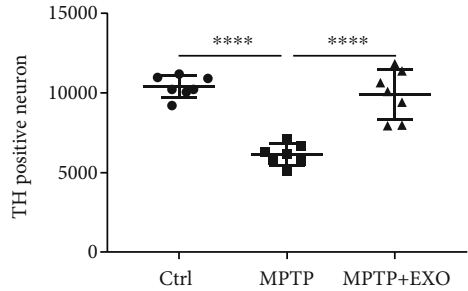

(b)

)

(c)
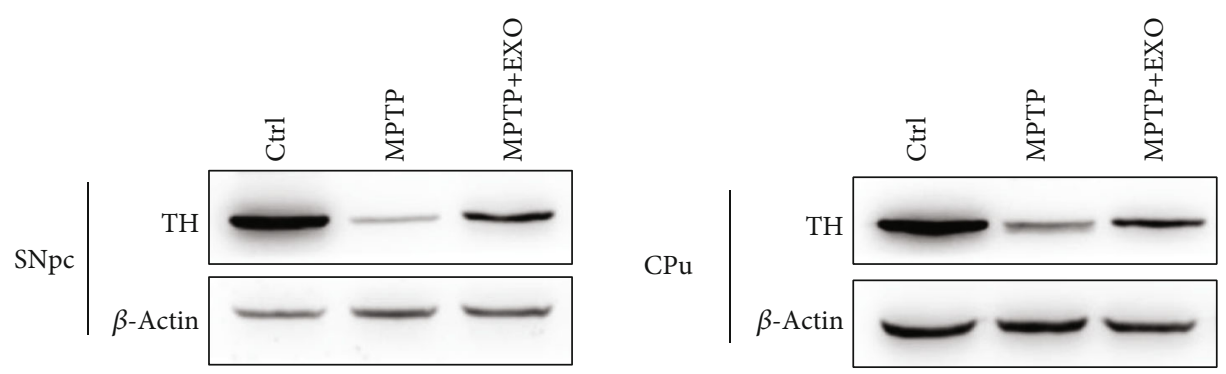

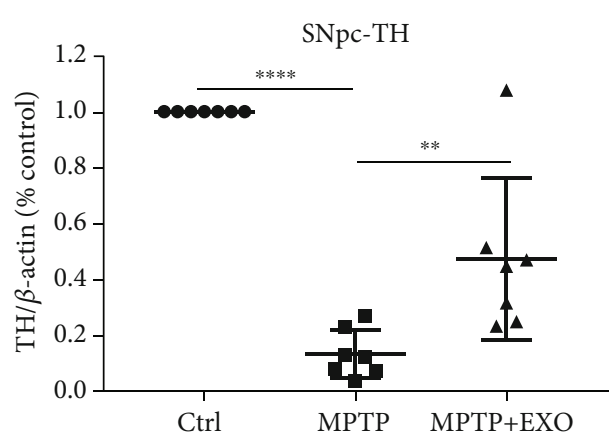

(d)
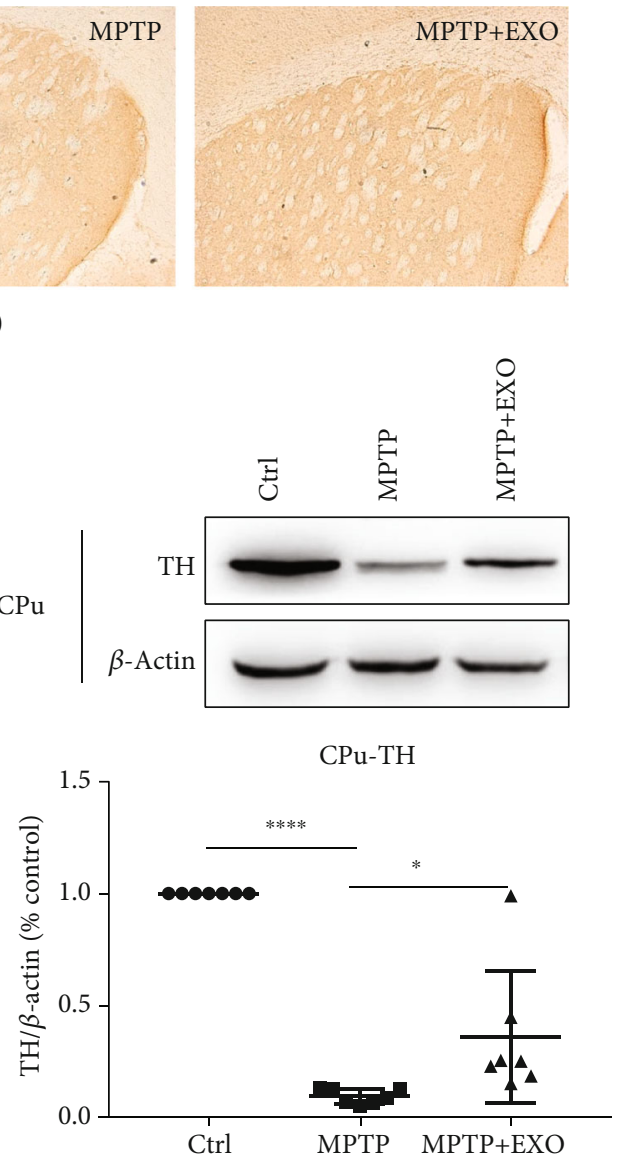

(e)

FIGURE 4: Exosomes prevent tyrosine hydroxylase (TH) loss in SNpc and CPu. Representative photomicrographs of TH-positive neurons in the SNpc region (a), quantitation of TH-positive neurons in SNpc (b), and representative photomicrographs of TH-positive neurons in the $\mathrm{CPu}$ region (c). Representative immunoblots and quantification of $\mathrm{TH}$ in the $\mathrm{SNpc}(\mathrm{d})$ and in the $\mathrm{CPu}(\mathrm{e})$. Error bars represent the means $\pm \mathrm{SD}, n=7,{ }^{*} p<0.05,{ }^{* *} p<0.01,{ }^{* * *} p<0.001$, and ${ }^{* * * *} p<0.0001$.

abnormal cell's faulty programming by transmitting the "correct" information to the abnormal cell. There is no doubt that exosomes participate in the development process of PD, and the mechanism may derive from two aspects: first, exosomes are directly involved in information transmission and transport; second, exosomes indirectly affect biochemical reactions by altering the level of metabolites.

Based on the above mechanism, researchers have been studying and evaluating the feasibility of exosomes as therapeutic agents. Some studies have indicated that exosomes from different sources play a considerable role in protecting cells and alleviating the disease process. Research has revealed that exosomes from dental pulp stem cells inhibit 6-hydroxydopamine-induced apoptosis of dopaminergic neurons [56]. Similar findings indicated that exosomes from neurons, embryonic stem cells, neural progenitor cells, and astrocytes protect neurons [57]. Many studies have yielded similar results to the above, but they were based on exosomes of one cell type. It is undeniable that communication and transmission in the body generally involve the interaction of various types of cells. In this study, we focused on the blood-derived exosomes, which included exosomes derived from multiple cells rather than just one type of cell [58]. This setting allows our "therapeutics" to contain more comprehensive information. Exosomes released by different nerve cells carry different information and play different roles. Our study revealed that blood exosomes extracted from healthy volunteers have a neuroprotective effect on MPTP- 




(a)

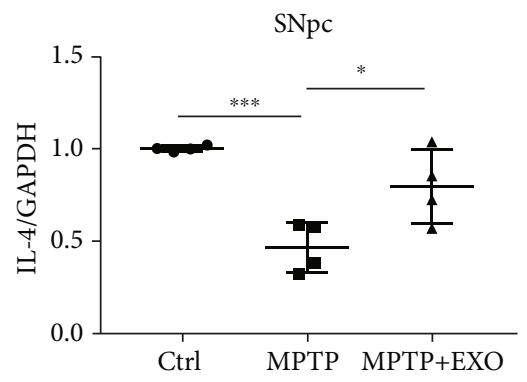

(d)

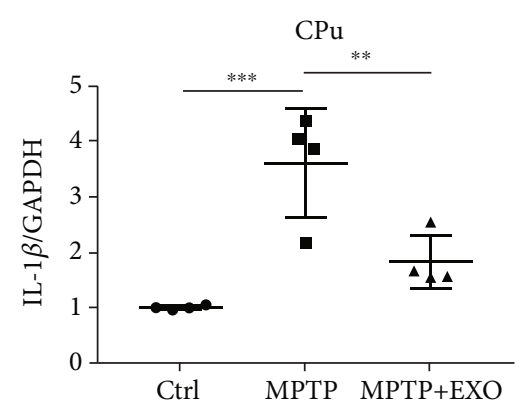

(g)

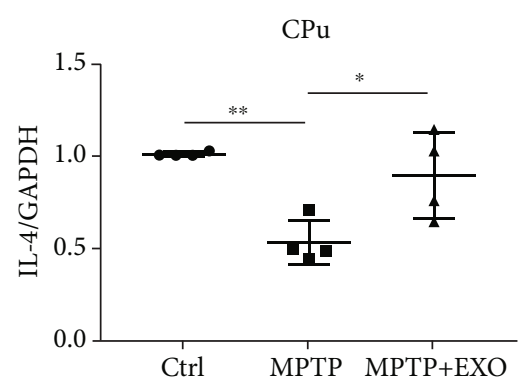

(j)

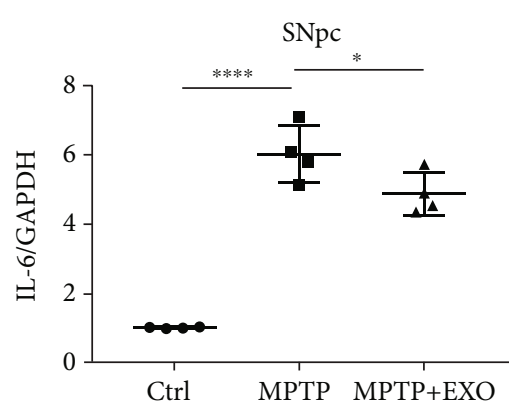

(b)

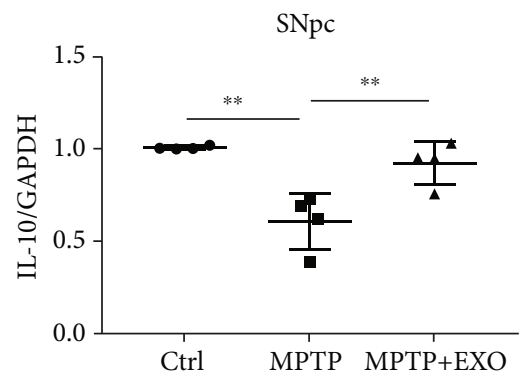

(e)

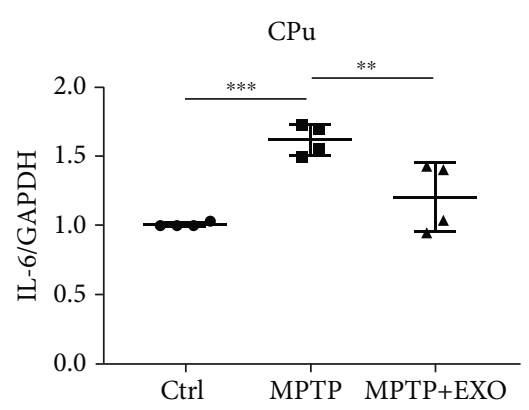

(h)

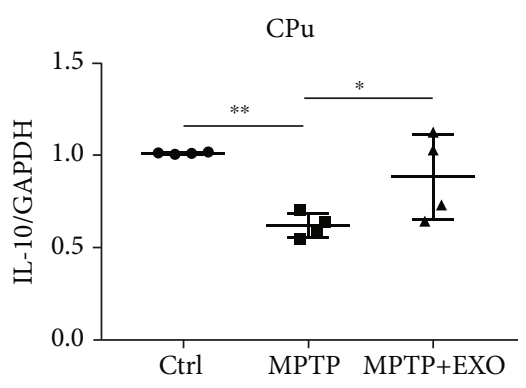

(k)

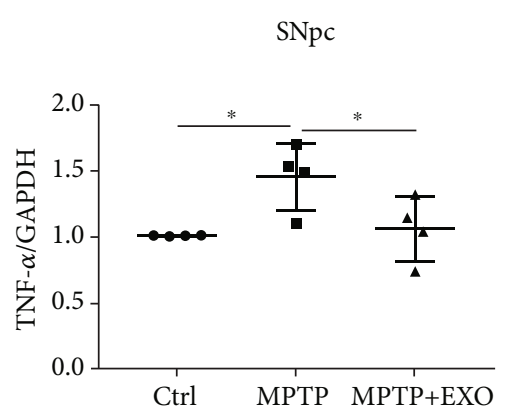

(c)

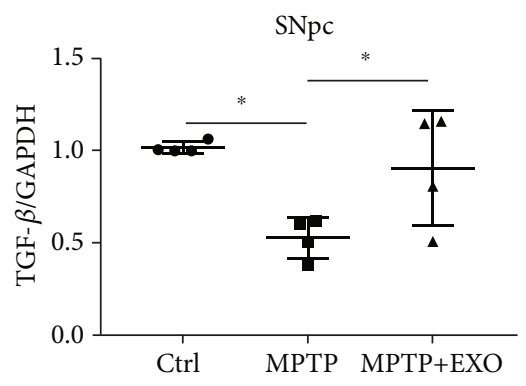

(f)

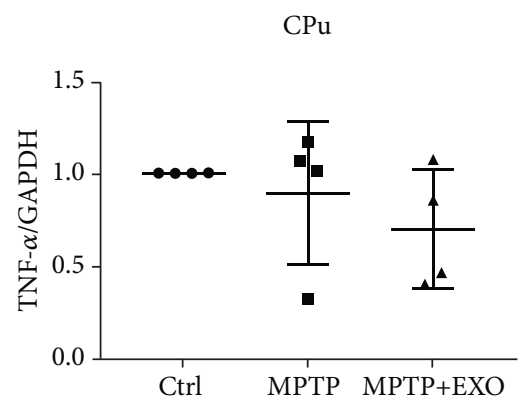

(i)

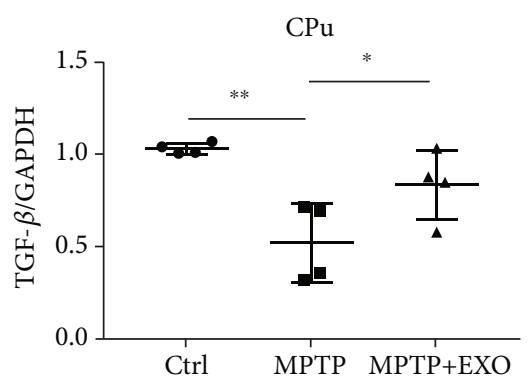

(1)

FIGURe 5: Exosomes attenuate neuroinflammation in SNpc and CPu. Data from qRT-PCR analyses of L- $1 \beta$ (a), IL-6 (b), TNF- $\alpha$ (c), IL-4 (d), IL-10 (e), TGF- $\beta$ (f) in SNpc, and L-1 $\beta$ (g), IL-6 (h), TNF- $\alpha$ (i), IL-4 (j), IL-10 (k), TGF- $\beta$ (l) in CPu. Error bars represent the means \pm SD, $n=4,{ }^{*} p<0.05,{ }^{* *} p<0.01,{ }^{* * *} p<0.001$, and ${ }^{* * * *} p<0.0001$.

treated mice. These protective effects were reflected by the restoration of the impaired motor coordination of mice, reduced loss of dopaminergic neurons, alleviated oxidative stress injury and neuroinflammation, and reduced cell apoptosis. We considered that the multiple neuroprotective effects in this study may be related to the source of exosomes, that is, blood exosomes are a complex composition comprising exosomes from a variety of nerve cells. There are two possible reasons for the therapeutic effects of blood exosomes.
First, we can consider that some of the information carried by blood exosomes originates in the brain, for example, exosomes released by neurons cross the blood-brain barrier to the blood. Some of the information is consistent between the blood and the brain. Second, we speculate that intravenously injected exosomes entered the brain through the blood-brain barrier, altered the original proportion of exosomes in the brain, and successfully affected the physiological and pathological processes of the treated mice. The studies by 


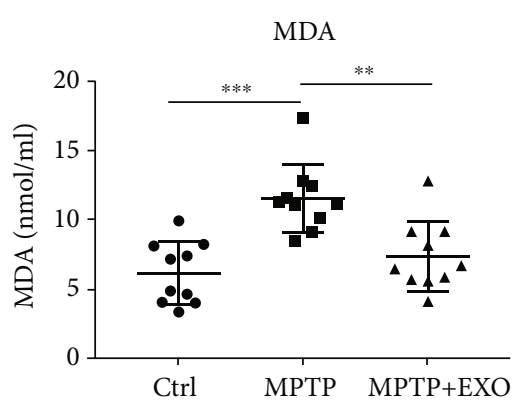

(a)

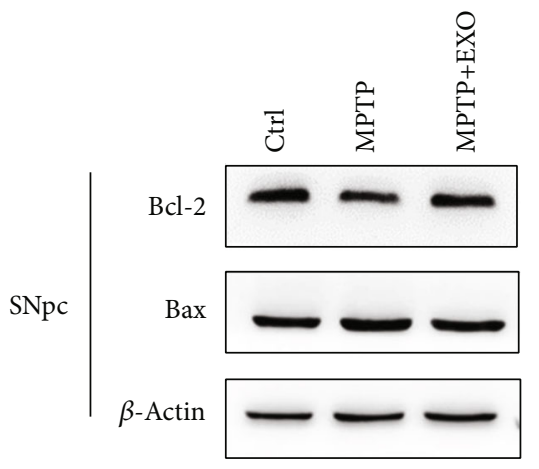

(c)

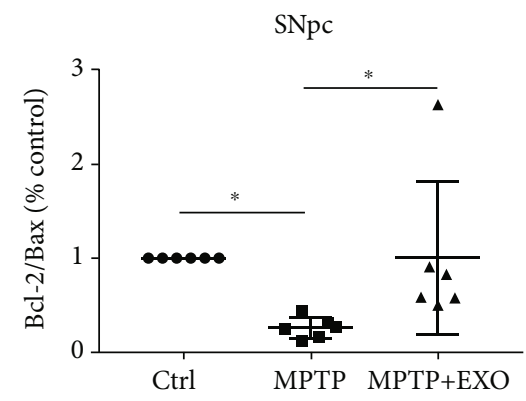

(e)

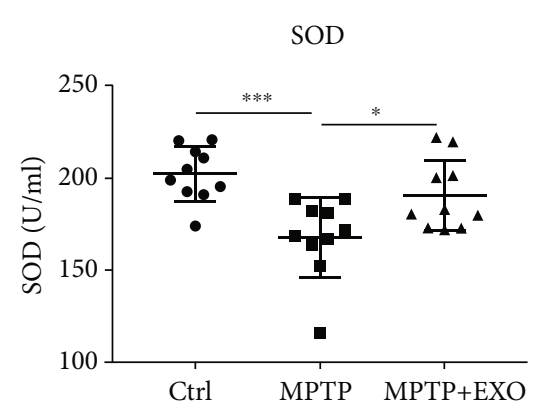

(b)

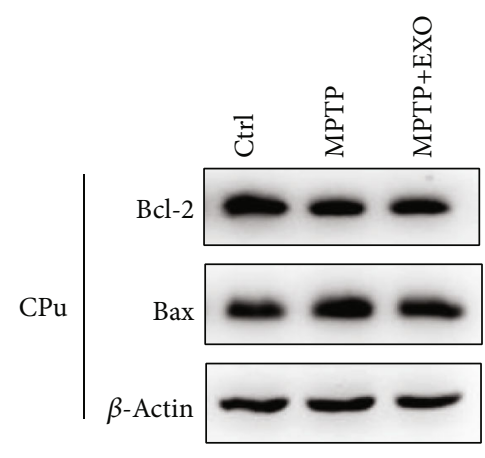

(d)

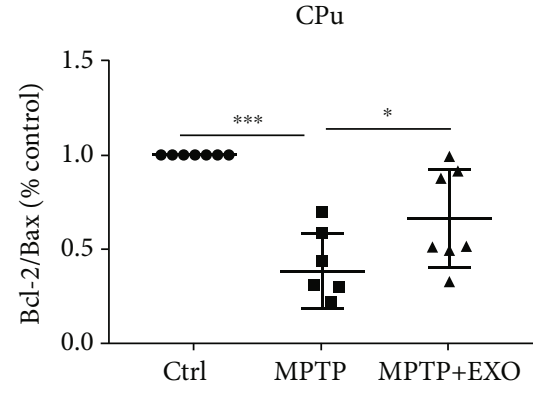

(f)

FIGURE 6: Exosomes alleviate oxidative stress injury in serum and the apoptosis in both SNpc and $\mathrm{CPu}$. Effects of exosome treatment on the changes of MDA (a) and total SOD (b), $n=10$. Representative bands of Bcl-2, Bax, and quantitative analysis results of Bcl-2/Bax in SNpc (c, e) and $\mathrm{Cpu}(\mathrm{d}, \mathrm{f}), n=7$. All values are means $\pm \mathrm{SD},{ }^{*} p<0.05,{ }^{* *} p<0.01$, and ${ }^{* * *} p<0.001$.

Qu et al. [59] and Peter et al. [60] provide strong support for this inference. They found that intravenous or intranasal injection of exosomes was targeted in relevant brain regions. Our results have suggested that exosomes are a promising target for the treatment of PD.

At present, the diagnosis of PD mainly depends on the assessment of motor symptoms according to the UK Parkinson's Disease Society Brain Bank clinical diagnostic criteria [61] and the patient's response to dopaminergic drugs. The degeneration of dopaminergic neurons before the onset of PD symptoms lasts several decades [62]. Therefore, identifying biomarkers of preclinical PD is the key to treat and predict this disease and distinguish it from other diseases with the same manifestation. After years of failing to find available biomarkers in metabolites of body fluids such as blood or urine, the focus has turned to exosomes in the hope of gaining more useful information. Recent studies have suggested $\alpha$-synuclein in plasma neuronal exosomes as a promising biomarker for the early diagnosis and prognosis of PD [63,
64]. In this study, widely-targeted metabolomics analysis was used to analyze the metabolite differences in exosomes in the serum and brain between healthy mice and MPTPtreated mice and explore the potential biomarkers in exosomes and the potential mechanism underlying exosome involvement in PD. Given the significant differences between MPTP mice and controls regarding the level of the 25 metabolites in the serum and brain, it was necessary to explore the potential of serum metabolites as biomarkers to differentiate between control and MPTP mice. We performed ROC curve analysis using the nine metabolites that contributed most to the differentiation of MPTP mice and controls. The data from our study suggested that nine exosomal metabolites, which significantly differed in both the brain and the serum between groups, exhibited excellent performance in differentiating PD and control mice. These metabolites included phenylacetate, (S)-(-)-2-hydroxyisocaproic acid, D-glucono-1,5lactone, cholesterol, triethyl phosphate, 1,2-dichloroethane, 2,4,6-trimethylphenol, and terpinolene (AUC > 90\%). 
Furthermore, 1,2-dichloroethane and (S)-(-)-2-hydroxyisocaproic acid have the ability to completely distinguish $\mathrm{PD}$ mice from control mice (AUC $=100 \%$ ). Our study differs from previous reports in that we synchronously detected exosomal metabolites in the brain and serum. The advantage of this study was that the metabolites of serum exosomes could reflect the metabolites of brain exosomes, which can be difficult to acquire from clinical patients. Thus, these eight metabolites are potential clinical PD biomarkers.

However, the limitation of this study lies in the absence of a detailed mechanism for the treatment of PD by exosomes. Further research should focus on different metabolites or RNAs in exosomes, which can act as specific targets, to reveal the underlying mechanism of $\mathrm{PD}$, provide feasible treatment options, and identify more accurate biomarkers.

\section{Conclusion}

In conclusion, through a series of experiments in behavior, pathology, and molecular biology, we confirmed that blood exosomes have protective effects on MPTP-treated PD model mice. This reflects the involvement of exosomes in the pathogenesis of neuron growth, oxidative stress, and neuroinflammation in PD. In addition, the widely-targeted metabolomics analysis revealed alteration of exosome metabolites and suggested the possibility of exosomes as potential biomarkers of PD. Our research provides novel suggestions for the treatment and prediction of PD.

\section{Data Availability}

All data generated or analyzed during this study are included in the manuscript.

\section{Ethical Approval}

Animal procedures were approved by the Animal Care and Use Committee of the Minzu University of China.

\section{Conflicts of Interest}

The authors declare no conflicts of interest.

\section{Authors' Contributions}

YC conceived and designed the experiments. TS, ZXD, and $\mathrm{XL}$ performed the experiments and analyzed the data. TS drafted the manuscript. YC and QSL reviewed and edited the manuscript. All authors read and approved the final manuscript.

\section{Acknowledgments}

This study was supported by the National Natural Science Foundation of China (81703492), Beijing Natural Science Foundation (7182092), and the MUC 111 project.

\section{Supplementary Materials}

Supplementary Figure 1:2D and 3D PCA Plots indicated different metabolic characteristics in serum and brain tissue. Supplementary methods: the blood exosome isolation and validation methods. Supplementary Table 1: information of healthy volunteers. Supplementary Table 2: 69 differentially expressed metabolites in serum. Supplementary Table 3: 148 differentially expressed metabolites in the brain. (Supplementary materials)

\section{References}

[1] M. Delenclos, D. R. Jones, P. J. McLean, and R. J. Uitti, "Biomarkers in Parkinson's disease: Advances and strategies," Parkinsonism \& Related Disorders, vol. 22, pp. S106-S110, 2016.

[2] A. Lee and R. M. Gilbert, "Epidemiology of Parkinson disease," Neurologic Clinics, vol. 34, no. 4, pp. 955-965, 2016.

[3] T. Pringsheim, N. Jette, A. Frolkis, and T. D. L. Steeves, "The prevalence of Parkinson's disease: a systematic review and meta-analysis," Movement Disorders, vol. 29, no. 13, pp. 1583-1590, 2014.

[4] G. E. Gillies, I. S. Pienaar, S. Vohra, and Z. Qamhawi, "Sex differences in Parkinson's disease," Frontiers in Neuroendocrinology, vol. 35, no. 3, pp. 370-384, 2014.

[5] L. V. Kalia and A. E. Lang, "Parkinson's disease," Lancet, vol. 386, no. 9996, pp. 896-912, 2015.

[6] R. Balestrino and A. H. V. Schapira, "Parkinson disease," European Journal of Neurology, vol. 27, no. 1, pp. 27-42, 2019.

[7] C. W. Olanow and J. A. Obeso, "The significance of defining preclinical or prodromal Parkinson's disease," Movement Disorders, vol. 27, no. 5, pp. 666-669, 2012.

[8] R. Cacabelos, "Parkinson's Disease: From Pathogenesis to Pharmacogenomics," International Journal of Molecular Sciences, vol. 18, no. 3, p. 551, 2017.

[9] A. Ascherio and M. A. Schwarzschild, "The epidemiology of Parkinson's disease: risk factors and prevention," Lancet Neurology, vol. 15, no. 12, pp. 1257-1272, 2016.

[10] M. A. Nalls, International Parkinson's Disease Genomics Consortium (IPDGC), N. Pankratz et al., "Large-scale metaanalysis of genome-wide association data identifies six new risk loci for Parkinson's disease," Nature Genetics, vol. 46, no. 9, pp. 989-993, 2014.

[11] V. Calabrese, A. Santoro, D. Monti et al., "Aging and Parkinson's disease: inflammaging, neuroinflammation and biological remodeling as key factors in pathogenesis," Free Radical Biology \& Medicine, vol. 115, pp. 80-91, 2018.

[12] M. Schwartz and J. Kipnis, "A common vaccine for fighting neurodegenerative disorders: recharging immunity for homeostasis," Trends in Pharmacological Sciences, vol. 25, no. 8, pp. 407-412, 2004.

[13] M. G. Tansey and M. S. Goldberg, "Neuroinflammation in Parkinson's disease: its role in neuronal death and implications for therapeutic intervention," Neurobiology of Disease, vol. 37, no. 3, pp. 510-518, 2010.

[14] S. Phani, J. D. Loike, and S. Przedborski, "Neurodegeneration and Inflammation in Parkinson's disease," Parkinsonism \& Related Disorders, vol. 18, pp. S207-S209, 2012.

[15] H. M. Gao and J. S. Hong, "Why neurodegenerative diseases are progressive: uncontrolled inflammation drives disease 
progression," Trends in Immunology, vol. 29, no. 8, pp. 357365, 2008.

[16] W. Le, J. Wu, and Y. Tang, "Protective Microglia and Their Regulation in Parkinson's Disease," Frontiers in Molecular Neuroscience, vol. 9, 2016.

[17] B. G. Trist, D. J. Hare, and K. L. Double, "Oxidative stress in the aging substantia nigra and the etiology of Parkinson's disease," Aging Cell, vol. 18, no. 6, article e13031, 2019.

[18] I. Ferrer, A. Martinez, R. Blanco, E. Dalfó, and M. Carmona, "Neuropathology of sporadic Parkinson disease before the appearance of parkinsonism: preclinical Parkinson disease," Journal of Neural Transmission (Vienna), vol. 118, no. 5, pp. 821-839, 2011.

[19] S. Le Saux, H. Aarrass, J. Lai-Kee-Him et al., "Post-production modifications of murine mesenchymal stem cell (mMSC) derived extracellular vesicles (EVs) and impact on their cellular interaction," Biomaterials, vol. 231, p. 119675, 2020.

[20] G. Van Niel, G. D'angelo, and G. Raposo, "Shedding light on the cell biology of extracellular vesicles," Nature Reviews. Molecular Cell Biology, vol. 19, no. 4, pp. 213-228, 2018.

[21] J. Fauré, G. Lachenal, M. Court et al., "Exosomes are released by cultured cortical neurones," Molecular and Cellular Neurosciences, vol. 31, no. 4, pp. 642-648, 2006.

[22] I. Potolicchio, G. J. Carven, X. Xu et al., "Proteomic analysis of microglia-derived exosomes: metabolic role of the aminopeptidase CD13 in neuropeptide catabolism," Journal of Immunology, vol. 175, no. 4, pp. 2237-2243, 2005.

[23] A. R. Taylor, M. B. Robinson, D. J. Gifondorwa, M. Tytell, and C. E. Milligan, "Regulation of heat shock protein 70 release in astrocytes: role of signaling kinases," Developmental Neurobiology, vol. 67, no. 13, pp. 1815-1829, 2007.

[24] R. M. Johnstone, "Revisiting the road to the discovery of exosomes," Blood Cells, Molecules, and Diseases, vol. 34, no. 3, pp. 214-219, 2005.

[25] G. Raposo and W. Stoorvogel, "Extracellular vesicles: exosomes, microvesicles, and friends," The Journal of Cell Biology, vol. 200, no. 4, pp. 373-383, 2013.

[26] E. Emmanouilidou, K. Melachroinou, T. Roumeliotis et al., "Cell-produced alpha-synuclein is secreted in a calciumdependent manner by exosomes and impacts neuronal survival," The Journal of Neuroscience, vol. 30, no. 20, pp. 68386851, 2010.

[27] C. Porro, M. A. Panaro, D. D. Lofrumento, E. Hasalla, and T. Trotta, "The multiple roles of exosomes in Parkinson's disease: an overview," Immunopharmacology and Immunotoxicology, vol. 41, no. 4, pp. 469-476, 2019.

[28] J. Howitt and A. F. Hill, "Exosomes in the Pathology of Neurodegenerative Diseases," Journal of Biological Chemistry, vol. 291, no. 52, pp. 26589-26597, 2016.

[29] M. D’Anca, C. Fenoglio, M. Serpente et al., "Exosome determinants of physiological aging and age-related neurodegenerative diseases," Frontiers in Aging Neuroscience, vol. 11, 2019.

[30] X. Wu, T. Zheng, and B. Zhang, "Exosomes in Parkinson's disease," Neuroscience Bulletin, vol. 33, no. 3, pp. 331-338, 2017.

[31] M. Shi, C. Liu, T. J. Cook et al., "Plasma exosomal $\alpha$-synuclein is likely CNS-derived and increased in Parkinson's disease," Acta Neuropathologica, vol. 128, no. 5, pp. 639-650, 2014.

[32] A. Stuendl, M. Kunadt, N. Kruse et al., "Induction of $\alpha$-synuclein aggregate formation by CSF exosomes from patients with Parkinson's disease and dementia with Lewy bodies," Brain : a journal of neurology, vol. 139, no. 2, pp. 481-494, 2016.
[33] H. Yu, T. Sun, J. An et al., "Potential roles of exosomes in Parkinson's disease: from pathogenesis, diagnosis, and treatment to prognosis," Frontiers in cell and developmental biology, vol. 8, p. 86, 2020.

[34] Z. G. Zhang and M. Chopp, "Exosomes in stroke pathogenesis and therapy," The Journal of Clinical Investigation, vol. 126, no. 4, pp. 1190-1197, 2016.

[35] Z. G. Zhang, B. Buller, and M. Chopp, "Exosomes - beyond stem cells for restorative therapy in stroke and neurological injury," Nature Reviews. Neurology, vol. 15, no. 4, pp. 193 203, 2019.

[36] Y. Song, Z. Li, T. He et al., "M2 microglia-derived exosomes protect the mouse brain from ischemia-reperfusion injury via exosomal miR-124," Theranostics, vol. 9, no. 10, pp. 29102923, 2019.

[37] Y. Zhang, M. Chopp, Y. Meng et al., "Effect of exosomes derived from multipluripotent mesenchymal stromal cells on functional recovery and neurovascular plasticity in rats after traumatic brain injury," Journal of Neurosurgery, vol. 122, no. 4, pp. 856-867, 2015.

[38] S. Huang, X. Ge, J. Yu et al., "Increased miR-124-3p in microglial exosomes following traumatic brain injury inhibits neuronal inflammation and contributes to neurite outgrowthviatheir transfer into neurons," The FASEB Journal, vol. 32, no. 1, pp. 512-528, 2017.

[39] M. Ding, Y. Shen, P. Wang et al., "Exosomes isolated from human umbilical cord mesenchymal stem cells alleviate neuroinflammation and reduce amyloid-beta deposition by modulating microglial activation in Alzheimer's disease," Neurochemical Research, vol. 43, no. 11, pp. 2165-2177, 2018.

[40] Z. X. Wei, G. J. Xie, X. Mao et al., "Exosomes from patients with major depression cause depressive-like behaviors in mice with involvement of miR-139-5p-regulated neurogenesis," Neuropsychopharmacology, vol. 45, no. 6, pp. 1050-1058, 2020.

[41] Y. Du, Y. Yu, Y. Hu et al., "Genome-wide, integrative analysis implicates exosome-derived microRNA dysregulation in schizophrenia," Schizophrenia Bulletin, vol. 45, no. 6, pp. 1257-1266, 2019.

[42] L. J. Vella, B. J. Scicluna, L. Cheng et al., "A rigorous method to enrich for exosomes from brain tissue," J Extracell Vesicles, vol. 6, no. 1, p. 1348885, 2017.

[43] S. Kim, S.-H. Kwon, T.-I. Kam et al., "Transneuronal Propagation of Pathologic $\alpha$-Synuclein from the Gut to the Brain Models Parkinson's Disease," Neuron, vol. 103, no. 4, pp. 627-641.e7, 2019.

[44] J. Havelund, N. Heegaard, N. Færgeman, and J. Gramsbergen, "Biomarker Research in Parkinson's Disease Using Metabolite Profiling," Metabolites, vol. 7, no. 3, p. 42, 2017.

[45] M. Kori, B. Aydın, S. Unal, K. Y. Arga, and D. Kazan, "Metabolic biomarkers and neurodegeneration: a pathway enrichment analysis of Alzheimer's disease, Parkinson's disease, and amyotrophic lateral sclerosis," OMICS, vol. 20, no. 11, pp. 645-661, 2016.

[46] Y. Shao and W. Le, "Recent advances and perspectives of metabolomics-based investigations in Parkinson's disease," Molecular Neurodegeneration, vol. 14, no. 1, p. 3, 2019.

[47] M. H. A. Bakar, C. K. Kai, W. N. W. Hassan, M. R. Sarmidi, H. Yaakob, and H. Z. Huri, "Mitochondrial dysfunction as a central event for mechanisms underlying insulin resistance: the roles of long chain fatty acids," Diabetes/Metabolism Research and Reviews, vol. 31, no. 5, pp. 453-475, 2015. 
[48] M. Trupp, P. Jonsson, A. Öhrfelt et al., "Metabolite and peptide levels in plasma and CSF differentiating healthy controls from patients with newly diagnosed Parkinson's disease," Journal of Parkinson's Disease, vol. 4, no. 3, pp. 549-560, 2014.

[49] P. A. LeWitt, J. Li, M. Lu, L. Guo, and P. Auinger, "Metabolomic biomarkers as strong correlates of Parkinson disease progression," Neurology, vol. 88, no. 9, pp. 862-869, 2017.

[50] T. Hatano, S. Saiki, A. Okuzumi, R. P. Mohney, and N. Hattori, "Identification of novel biomarkers for Parkinson's disease by metabolomic technologies," Journal of Neurology, Neurosurgery, and Psychiatry, vol. 87, no. 3, pp. 295-301, 2016.

[51] H. Luan, L.-F. Liu, N. Meng et al., "LC-MS-Based Urinary Metabolite Signatures in Idiopathic Parkinson's Disease," Journal of Proteome Research, vol. 14, no. 1, pp. 467-478, 2014.

[52] L. Vella, A. Hill, and L. Cheng, "Focus on extracellular vesicles: exosomes and their role in protein trafficking and biomarker potential in Alzheimer's and Parkinson's disease," International Journal of Molecular Sciences, vol. 17, no. 2, p. 173, 2016.

[53] R. Ghidoni, L. Benussi, and G. Binetti, "Exosomes: the Trojan horses of neurodegeneration," Medical Hypotheses, vol. 70, no. 6, pp. 1226-1227, 2008.

[54] B. M. Coleman and A. F. Hill, "Extracellular vesicles - their role in the packaging and spread of misfolded proteins associated with neurodegenerative diseases," Seminars in Cell \& Developmental Biology, vol. 40, pp. 89-96, 2015.

[55] C. Han, N. Xiong, X. Guo et al., "Exosomes from patients with Parkinson's disease are pathological in mice," Journal of Molecular Medicine, vol. 97, no. 9, pp. 1329-1344, 2019.

[56] A. Jarmalavičiūtè, V. Tunaitis, U. Pivoraite, A. Venalis, and A. Pivoriūnas, "Exosomes from dental pulp stem cells rescue human dopaminergic neurons from 6-hydroxy-dopamineinduced apoptosis," Cytotherapy, vol. 17, no. 7, pp. 932-939, 2015.

[57] M. Deng, H. Xiao, H. Peng et al., "Preservation of neuronal functions by exosomes derived from different human neural cell types under ischemic conditions," The European Journal of Neuroscience, vol. 47, no. 2, pp. 150-157, 2018.

[58] T. Ohmichi, M. Mitsuhashi, H. Tatebe, T. Kasai, O. M. Ali elAgnaf, and T. Tokuda, "Quantification of brain-derived extracellular vesicles in plasma as a biomarker to diagnose Parkinson's and related diseases," Parkinsonism \& Related Disorders, vol. 61, pp. 82-87, 2019.

[59] M. Qu, Q. Lin, L. Huang et al., "Dopamine-loaded blood exosomes targeted to brain for better treatment of Parkinson's disease," Journal of controlled release : official journal of the Controlled Release Society, vol. 287, pp. 156-166, 2018.

[60] N. Perets, O. Betzer, R. Shapira et al., "Golden exosomes selectively target brain pathologies in neurodegenerative and neurodevelopmental disorders," Nano Letters, vol. 19, no. 6, pp. 3422-3431, 2019.

[61] W. R. Gibb and A. J. Lees, "The relevance of the Lewy body to the pathogenesis of idiopathic Parkinson's disease," Journal of Neurology, Neurosurgery, and Psychiatry, vol. 51, no. 6, pp. 745-752, 1988.

[62] R. B. Postuma, D. Aarsland, P. Barone et al., "Identifying prodromal Parkinson's disease: pre-motor disorders in Parkinson's disease," Movement Disorders, vol. 27, no. 5, pp. 617626, 2012.
[63] S. Lemprière, "Exosomal $\alpha$-synuclein as a biomarker for Parkinson disease," Nature Reviews Neurology, vol. 16, no. 5, pp. 242-243, 2020.

[64] M. Niu, Y. Li, G. Li et al., "A longitudinal study on $\alpha$-synuclein in plasma neuronal exosomes as a biomarker for Parkinson's disease development and progression," European Journal of Neurology, vol. 27, no. 6, pp. 967-974, 2020. 